Sharif University of Technology
Scientia Iranica
Transactions E: Industrial Engineering
hCIENTIA

\title{
Efficient ratio-type estimators of finite population mean based on correlation coefficient
}

\author{
M. Irfan ${ }^{a, b, *}$, M. Javed ${ }^{a, b}$, and Zh. $\operatorname{Lin}^{a}$ \\ a. Department of Mathematics, Institute of Statistics, Zhejiang University, Hangzhou 310027, China. \\ b. Department of Statistics, Government College University, Faisalabad, Pakistan.
}

Received 28 September 2016; received in revised form 4 February 2017; accepted 20 May 2017

\author{
KEYWORDS \\ Auxiliary variable; \\ Bias; \\ Correlation coefficient; \\ Efficiency; \\ Mean squared error; \\ Ratio-type estimators.
}

\begin{abstract}
We proposed efficient families of ratio-type estimators to estimate finite population mean using known correlation coefficient between study variable and auxiliary variable by adopting Singh and Tailor's [Singh, H.P., and Tailor, R. "Use of known correlation coefficient in estimating the finite population means", Statistics in Transition, 6(4), pp. 555-560 (2003)] estimator and Kadilar and Cingi's [Kadilar, C., and Cingi, H. "An improvement in estimating the population mean by using the correlation coefficient", Hacettepe Journal of Mathematics and Statistics, 35(1) pp. 103-109 (2006a)] class of estimators in simple random sampling without replacement. The newly proposed estimators behaved efficiently as compared to the common unbiased estimator, traditional ratio estimator, and the other competing estimators. Bias, mean squared error, and minimum mean squared error of the proposed ratio-type estimators were derived. Moreover, theoretical findings were proven with cooperation of two real data sets.
\end{abstract}

(C) 2018 Sharif University of Technology. All rights reserved.

\section{Introduction}

The ratio estimator suggested by Cochran [1] plays an important role in the scenario of positive (high) correlation among study and auxiliary variables. One of the hottest issues in the theory of sample survey is the estimation of finite population mean with different sampling techniques. Extensive work has been done on this issue using auxiliary information, such as population median, mean, quartiles, deciles, coefficient of variation, coefficient of correlation, coefficient of skewness, coefficient of kurtosis, etc. Various estimators or classes of estimators have been proposed by several authors, including Sisodia and Dwivedi [2], Upadhyaya and Singh [3], Singh and Tailor [4], Kadilar and

\footnotetext{
*. Corresponding author.

E-mail addresses: mirfan@zju.edu.cn (M. Irfan);

mariastat@zju.edu.cn (M. Javed); zlin@zju.edu.cn (Zh. Lin)
}

doi: $10.24200 /$ sci.2017.4455
Cingi [5,6], Singh et al. [7,8], Gupta and Shabbir [9], Koyuncu and Kadilar [10,11], Yan and Tian [12], Haq and Shabbir [13], Singh and Solanki [14], Yadav and Kadilar [15], Subramani and Kumarapandiyan [16, 17], Subramani and Prabavathy [18], Yadav et al. [19], Khan et al. [20], Kumar [21], Irfan et al. [22], Walia et al. [23] etc., as solutions to this issue to get improved results.

Let " $N$ " be the population size and " $n$ " be the sample size such that $n<N$ is drawn under simple random sampling without replacement (SRSWOR) scheme. Assume $y$ and $x$ are the study and auxiliary variables, respectively. Here, $y_{i}$ and $x_{i}$ represent the values of $y$ and $x$ for the $i$ th unit of the population.

Some important formulae used in this manuscript are given as follows:

Population mean of study variable:

$$
\bar{Y}=N^{-1} \sum_{i=1}^{N} y_{i}
$$


Population mean of auxiliary variable:

$$
\bar{X}=N^{-1} \sum_{i=1}^{N} x_{i} .
$$

Sample mean of study variable:

$$
\bar{y}=n^{-1} \sum_{i=1}^{n} y_{i}
$$

Sample mean of auxiliary variable:

$$
\bar{x}=n^{-1} \sum_{i=1}^{n} x_{i}
$$

Sampling fraction:

$$
f=\frac{n}{N} \text {. }
$$

Ratio of population mean of study variable to population mean of auxiliary variable:

$$
R=\frac{\bar{Y}}{\bar{X}} .
$$

Constant term:

$$
\theta=\left(\frac{1}{n}-\frac{1}{N}\right)
$$

Relative error terms and their expectations have been defined in order to get the bias, Mean Squared Error (MSE), and minimum MSE for the existing and proposed ratio-type estimators in this way.

Let $\xi_{0}=\frac{\bar{y}-\bar{Y}}{\bar{Y}}$ and $\xi_{1}=\frac{\bar{x}-\bar{X}}{\bar{X}}$ such that $E\left(\xi_{i}\right)=0$ for $i=0$ and 1 , where $E($.$) represents the mathematical$ expectation and:

$$
E\left(\xi_{0}^{2}\right)=\theta \frac{S_{y}^{2}}{\bar{Y}^{2}}, \quad E\left(\xi_{1}^{2}\right)=\theta \frac{S_{x}^{2}}{\bar{X}^{2}}, \quad E\left(\xi_{0} \xi_{1}\right)=\theta \frac{S_{y x}}{\bar{Y} \bar{X}},
$$

where:

$$
\begin{aligned}
& S_{y}^{2}=(N-1)^{-1} \sum_{i=1}^{N}\left(y_{i}-\bar{Y}\right)^{2}, \\
& S_{x}^{2}=(N-1)^{-1} \sum_{i=1}^{N}\left(x_{i}-\bar{X}\right)^{2}, \\
& S_{y x}=(N-1)^{-1} \sum_{i=1}^{N}\left(y_{i}-\bar{Y}\right)\left(x_{i}-\bar{X}\right) .
\end{aligned}
$$

The motivation behind this manuscript is to develop new efficient families of ratio-type estimators to estimate population mean using SRSWOR scheme with the help of known correlation coefficient between study variable and auxiliary variable. In the following, some well-known traditional and existing estimators in SRSWOR are provided.

Commonly used unbiased estimator of $\bar{Y}$ is:

$$
\hat{\bar{Y}}=\bar{y} \text {. }
$$

Estimator in Eq. (1) has the following mean squared error or variance:

$$
\operatorname{MSE}(\hat{\bar{Y}})=V(\hat{\bar{Y}})=\theta S_{y}^{2} .
$$

The traditional ratio estimator of population mean suggested by Cochran [1] is defined as:

$$
\hat{\bar{Y}}_{R}=\bar{y}\left(\frac{\bar{X}}{\bar{x}}\right), \quad \bar{x} \neq 0 .
$$

Bias and MSE of $\hat{\bar{Y}}_{R}$ up to the first degree of approximation are, respectively, given as:

$$
\operatorname{Bias}\left(\hat{\bar{Y}}_{R}\right) \approx \theta \bar{Y}\left[C_{x}^{2}-\rho_{y x} C_{y} C_{x}\right],
$$

and:

$$
\operatorname{MSE}\left(\hat{\bar{Y}}_{R}\right) \approx \theta \bar{Y}^{2}\left[C_{y}^{2}+C_{x}^{2}(1-2 \varphi)\right],
$$

where:

$$
\begin{aligned}
& \varphi=\rho_{y x} \frac{C_{y}}{C_{x}}, \quad C_{y}=\frac{S_{y}}{\bar{Y}}, \\
& C_{x}=\frac{S_{x}}{\bar{X}}, \quad \rho_{y x}=\left(S_{y} S_{x}\right)^{-1} S_{y x} .
\end{aligned}
$$

Given below is the introduction of some other competing ratio-type estimators that take known correlation coefficient between study and auxiliary variables into account.

\subsection{Singh and tailor estimator}

In order to estimate finite population mean, Singh and Tailor [4] proposed ratio estimator by utilizing the known correlation coefficient $\rho_{y x}$ :

$$
\hat{\bar{Y}}_{S T}=\bar{y}\left(\frac{\bar{X}+\rho_{y x}}{\bar{x}+\rho_{y x}}\right) .
$$

Bias and MSE up to the first order of approximation of $\hat{\bar{Y}}_{S T}$ in Eq. (6) are given by:

$$
\operatorname{Bias}\left(\hat{\bar{Y}}_{S T}\right) \approx \theta \bar{Y} \lambda\left[\lambda C_{x}^{2}-\rho_{y x} C_{y} C_{x}\right],
$$

and:

$$
\operatorname{MSE}\left(\hat{\bar{Y}}_{S T}\right) \approx \theta \bar{Y}^{2}\left[C_{y}^{2}+C_{x}^{2} \lambda(\lambda-2 \varphi)\right],
$$

where:

$$
\lambda=\frac{\bar{X}}{\bar{X}+\rho_{y x}} .
$$




\subsection{Kadilar and Cingi estimators}

Kadilar and Cingi [24] proposed a class of ratio estimators to estimate population mean under SRSWOR in the light of the work done by Upadhyaya and Singh [3] and Singh and Tailor [4]. Their proposed class of estimators is:

$$
\begin{aligned}
& \hat{\bar{Y}}_{K C 1}=\bar{y}\left(\frac{\bar{X} C_{x}+\rho_{y x}}{\bar{x} C_{x}+\rho_{y x}}\right), \\
& \hat{\bar{Y}}_{K C 2}=\bar{y}\left(\frac{\bar{X} \rho_{y x}+C_{x}}{\bar{x} \rho_{y x}+C_{x}}\right), \\
& \hat{\bar{Y}}_{K C 3}=\bar{y}\left(\frac{\bar{X} \beta_{2}(x)+\rho_{y x}}{\bar{x} \beta_{2}(x)+\rho_{y x}}\right), \\
& \hat{\bar{Y}}_{K C 4}=\bar{y}\left(\frac{\bar{X} \rho_{y x}+\beta_{2}(x)}{\bar{x} \rho_{y x}+\beta_{2}(x)}\right),
\end{aligned}
$$

where $\rho_{y x}, C_{x}$, and $\beta_{2}(x)$ are the correlation coefficient between study and auxiliary variables, coefficient of variation, and coefficient of kurtosis of the auxiliary variable, respectively.

Bias and MSE of the above-mentioned estimators in Eqs. (9) to (12) are listed below:

$$
\operatorname{Bias}\left(\hat{\bar{Y}}_{K C i}\right) \approx \theta \bar{Y} C_{x}^{2} \lambda_{i}\left(\lambda_{i}-\varphi\right), \quad i=1,2,3,4 .
$$

and:

$$
\begin{aligned}
& \operatorname{MSE}\left(\hat{\bar{Y}}_{K C i}\right) \approx \theta \bar{Y}^{2}\left[C_{y}^{2}+C_{x}^{2} \lambda_{i}\left(\lambda_{i}-2 \varphi\right)\right], \\
& i=1,2,3,4
\end{aligned}
$$

where:

$$
\begin{array}{ll}
\lambda_{1}=\frac{\bar{X} C_{x}}{\bar{X} C_{x}+\rho_{y x}}, \quad \lambda_{2}=\frac{\bar{X} \rho_{y x}}{\bar{X} \rho_{y x}+C_{x}}, \\
\lambda_{3}=\frac{\bar{X} \beta_{2}(x)}{\bar{X} \beta_{2}(x)+\rho_{y x}}, \quad \lambda_{4}=\frac{\bar{X} \rho_{y x}}{\bar{X} \rho_{y x}+\beta_{2}(x)} .
\end{array}
$$

Kadilar and Cingi [25] defined another class of ratiotype estimators as:

$$
\hat{\bar{Y}}_{K C i}^{*}=[\bar{y}+b(\bar{X}-\bar{x})] \alpha_{i}, \quad i=1,2,3,4,5,
$$

where:

$$
\begin{aligned}
& \alpha_{1}=\frac{\bar{X}+\rho_{y x}}{\bar{x}+\rho_{y x}}, \quad \alpha_{2}=\frac{\bar{X} C_{x}+\rho_{y x}}{\bar{x} C_{x}+\rho_{y x}}, \\
& \alpha_{3}=\frac{\bar{X} \rho_{y x}+C_{x}}{\bar{x} \rho_{y x}+C_{x}}, \quad \alpha_{4}=\frac{\bar{X} \beta_{2}(x)+\rho_{y x}}{\bar{x} \beta_{2}(x)+\rho_{y x}}, \\
& \alpha_{5}=\frac{\bar{X} \rho_{y x}+\beta_{2}(x)}{\bar{x} \rho_{y x}+\beta_{2}(x)}
\end{aligned}
$$

The following are the expressions for bias and MSE of the $\hat{\bar{Y}}_{K C i}^{*}$ estimators:

$$
\operatorname{Bias}\left(\hat{\bar{Y}}_{K C i}^{*}\right) \approx \theta \bar{Y} C_{x}^{2} \alpha_{i}^{* 2}, \quad i=1,2,3,4,5,
$$

and:

$$
\begin{gathered}
\operatorname{MSE}\left(\hat{\bar{Y}}_{K C i}^{*}\right) \approx \theta \bar{Y}^{2}\left[\alpha_{i}^{* 2} C_{x}^{2}+C_{y}^{2}\left(1-\rho_{y x}^{2}\right)\right], \\
i=1,2,3,4,5
\end{gathered}
$$

where:

$$
\begin{aligned}
& \alpha_{1}^{*}=\frac{\bar{X}}{\bar{X}+\rho_{y x}}, \quad \alpha_{2}^{*}=\frac{\bar{X} C_{x}}{\bar{X} C_{x}+\rho_{y x}}, \\
& \alpha_{3}^{*}=\frac{\bar{X} \rho_{y x}}{\bar{X} \rho_{y x}+C_{x}}, \quad \alpha_{4}^{*}=\frac{\bar{X} \beta_{2}(x)}{\bar{X} \beta_{2}(x)+\rho_{y x}}, \\
& \alpha_{5}^{*}=\frac{\bar{X} \rho_{y x}}{\bar{X} \rho_{y x}+\beta_{2}(x)} .
\end{aligned}
$$

\section{Proposed families of ratio-type estimators}

Inspired by Singh and Tailor [4] and Kadilar and Cingi $[24,25]$, we propose efficient families of ratiotype estimators, i.e., $\left(\hat{\bar{Y}}_{P i}\right.$, i.e., $\left.i=1,3,5, \ldots, 11\right)$ and $\left(\hat{\bar{Y}}_{P j}\right.$, i.e., $\left.j=2,4,6, \ldots, 16\right)$, to estimate population mean using the correlation coefficient between study and auxiliary variables under SRSWOR scheme.

\subsection{The first proposed family of ratio-type estimators}

$$
\begin{aligned}
& \hat{\bar{Y}}_{P 1}=k \bar{y}\left(\frac{\bar{X} C_{x}+\rho_{y x}}{\bar{x} C_{x}+\rho_{y x}}\right)^{\frac{\bar{x} C_{x}}{\bar{X} C_{x}+\rho_{y x}}}, \\
& \hat{\bar{Y}}_{P 3}=k \bar{y}\left(\frac{\bar{X} \beta_{2}(x)+\rho_{y x}}{\bar{x} \beta_{2}(x)+\rho_{y x}}\right)^{\frac{\bar{X} \beta_{2}(x)}{\bar{X} \beta_{2}(x)+\rho_{y x}}}, \\
& \hat{\bar{Y}}_{P 5}=k \bar{y}\left(\frac{\bar{X} \beta_{2}(x)+\rho_{y x} C_{x}}{\bar{x} \beta_{2}(x)+\rho_{y x} C_{x}}\right)^{\frac{\bar{X} \beta_{2}(x)}{\bar{X} \beta_{2}(x)+\rho_{y x} C_{x}}}, \\
& \hat{\bar{Y}}_{P 7}=k \bar{y}\left(\frac{\bar{X} \rho_{y x} \beta_{2}(x)+C_{x}}{\bar{x} \rho_{y x} \beta_{2}(x)+C_{x}}\right)^{\frac{\bar{x} \rho_{y x} \beta_{2}(x)}{\bar{X} \rho_{y x} \beta_{2}(x)+C_{x}}}, \\
& \hat{\bar{Y}}_{P 9}=k \bar{y}\left(\frac{\bar{X}+\rho_{y x}}{\bar{x}+\rho_{y x}}\right)^{\frac{\bar{x}}{\bar{X}+\rho_{y x}}} \\
& \hat{\bar{Y}}_{P 11}=k \bar{y}\left(\frac{\bar{X} C_{x} \beta_{2}(x)+\rho_{y x}}{\bar{x} C_{x} \beta_{2}(x)+\rho_{y x}}\right)^{\frac{\bar{X} C_{x} \beta_{2}(x)}{\bar{X} C_{x} \beta_{2}(x)+\rho_{y x}}},
\end{aligned}
$$

where $k$ is the selected appropriate constant whose value is determined later in Theorem 2.1 and $\bar{X}$ population mean, $C_{x}$ population coefficient of variation, $\rho_{y x}$ population correlation coefficient, and $\beta_{2}(x)$ population coefficient of kurtosis are the known parameters. 


\section{Theorem 2.1}

The properties of $\hat{\bar{Y}}_{P i}$, i.e., $i=1,3,5, \ldots, 11$ are:

1) $\operatorname{Bias}\left(\hat{\bar{Y}}_{P i}\right) \approx \bar{Y}(k-1)$

$$
+\theta \bar{Y} k\left[\frac{1}{2}\left(\psi_{i}^{4}+\psi_{i}^{3}\right) C_{x}^{2}-\psi_{i}^{2} \rho_{y x} C_{y} C_{x}\right],
$$

2) $\operatorname{MSE}\left(\hat{\bar{Y}}_{P i}\right) \approx \bar{Y}^{2}\left[\left(\frac{A_{1 i}}{A_{2 i}}-1\right)^{2}+\left(\frac{A_{1 i}}{A_{2 i}}\right)^{2}\left(E\left(\xi_{0}^{2}\right)\right.\right.$

$$
\begin{aligned}
& \left.+\left(2 \psi_{i}^{4}+\psi_{i}^{3}\right) E\left(\xi_{1}^{2}\right)-4 \psi_{i}^{2} E\left(\xi_{0} \xi_{1}\right)\right) \\
& \left.-\frac{A_{1 i}}{A_{2 i}}\left(\left(\psi_{i}^{4}+\psi_{i}^{3}\right) E\left(\xi_{1}^{2}\right)-2 \psi_{i}^{2} E\left(\xi_{0} \xi_{1}\right)\right)\right],
\end{aligned}
$$

3) $\operatorname{MSE}_{\min }\left(\hat{\bar{Y}}_{P i}\right) \approx \bar{Y}^{2}\left[1-\frac{A_{1 i}^{2}}{2 A_{2 i}}\right]$,

where:

$$
\begin{aligned}
& \psi_{1}=\frac{\bar{X} C_{x}}{\bar{X} C_{x}+\rho_{y x}}, \quad \psi_{3}=\frac{\bar{X} \beta_{2}(x)}{\bar{X} \beta_{2}(x)+\rho_{y x}}, \\
& \psi_{5}=\frac{\bar{X} \beta_{2}(x)}{\bar{X} \beta_{2}(x)+\rho_{y x} C_{x}}, \quad \psi_{7}=\frac{\bar{X} \rho_{y x} \beta_{2}(x)}{\bar{X} \rho_{y x} \beta_{2}(x)+C_{x}}, \\
& \psi_{9}=\frac{\bar{X}}{\bar{X}+\rho_{y x}}, \quad \psi_{11}=\frac{\bar{X} C_{x} \beta_{2}(x)}{\bar{X} C_{x} \beta_{2}(x)+\rho_{y x}} .
\end{aligned}
$$

\section{Proof}

To prove Eqs. (24)-(26), the proposed family of ratiotype estimators $\left(\hat{\bar{Y}}_{P i}\right.$, i.e., $\left.i=1,3,5, \ldots, 11\right)$ can be written in terms of $\xi_{i}^{\prime}$ s as:

$$
\hat{\bar{Y}}_{P i}=k \bar{Y}\left(1+\xi_{0}\right)\left[1+\psi_{i} \xi_{1}\right]^{-\psi_{i}},
$$

or:

$$
\hat{\bar{Y}}_{P i}=k \bar{Y}\left(1+\xi_{0}\right)\left[1-\psi_{i}^{2} \xi_{1}+\frac{1}{2} \psi_{i}^{3}\left(\psi_{i}+1\right) \xi_{1}^{2}\right] .
$$

Simplifying the R.H.S. of Eq. (28) up to the first degree of approximation and, then, subtracting $\bar{Y}$ from both sides, we get:

$$
\begin{aligned}
\hat{\bar{Y}}_{P i}-\bar{Y}=\bar{Y} & {\left[k+k \xi_{0}-k \psi_{i}^{2} \xi_{1}+\frac{1}{2} k\left(\psi_{i}^{4}+\psi_{i}^{3}\right) \xi_{1}^{2}\right.} \\
& \left.-k \psi_{i}^{2} \xi_{0} \xi_{1}-1\right] .
\end{aligned}
$$

Bias and the MSE of the proposed family of ratio-type estimators ( $\hat{\bar{Y}}_{P i}$, i.e., $\left.i=1,3,5, \ldots, 11\right)$ are, respectively, given as:

$$
\begin{aligned}
\operatorname{Bias}\left(\hat{\bar{Y}}_{P i}\right)= & E\left(\hat{\bar{Y}}_{P i}-\bar{Y}\right) \approx \bar{Y}(k-1) \\
& +\theta \bar{Y} k\left[\frac{1}{2}\left(\psi_{i}^{4}+\psi_{i}^{3}\right) C_{x}^{2}-\psi_{i}^{2} \rho_{y x} C_{y} C_{x}\right],
\end{aligned}
$$

and:

$$
\begin{aligned}
\operatorname{MSE}\left(\hat{\bar{Y}}_{P i}\right)= & \left(\hat{\bar{Y}}_{P i}-\bar{Y}\right)^{2} \approx \bar{Y}^{2}\left[(k-1)^{2}\right. \\
& +k^{2}\left(E\left(\xi_{0}^{2}\right)+\left(2 \psi_{i}^{4}+\psi_{i}^{3}\right) E\left(\xi_{1}^{2}\right)\right. \\
& \left.-4 \psi_{i}^{2} E\left(\xi_{0} \xi_{1}\right)\right)-k\left(\left(\psi_{i}^{4}+\psi_{i}^{3}\right) E\left(\xi_{1}^{2}\right)\right. \\
& \left.\left.-2 \psi_{i}^{2} E\left(\xi_{0} \xi_{1}\right)\right)\right] .
\end{aligned}
$$

To get the optimal value of $k$, differentiating Eq. (31) with respect to $k$ and equating to zero, we have:

$$
\begin{aligned}
& k=\frac{2+\left(\psi_{i}^{4}+\psi_{i}^{3}\right) E\left(\xi_{1}^{2}\right)-2 \psi_{i}^{2} E\left(\xi_{0} \xi_{1}\right)}{2+2\left[E\left(\xi_{0}^{2}\right)+\left(2 \psi_{i}^{4}+\psi_{i}^{3}\right) E\left(\xi_{1}^{2}\right)-4 \psi_{i}^{2} E\left(\xi_{0} \xi_{1}\right)\right]}, \\
& k=\frac{1+\frac{\theta}{2}\left[\left(\psi_{i}^{4}+\psi_{i}^{3}\right) C_{x}^{2}-2 \psi_{i}^{2} \rho_{y x} C_{y} C_{x}\right]}{1+\theta\left[C_{y}^{2}+\left(2 \psi_{i}^{4}+\psi_{i}^{3}\right) C_{x}^{2}-4 \psi_{i}^{2} \rho_{y x} C_{y} C_{x}\right]}, \\
& k=\frac{A_{1 i}}{A_{2 i}}
\end{aligned}
$$

where:

$$
A_{1 i}=1+\frac{\theta}{2}\left[\left(\psi_{i}^{4}+\psi_{i}^{3}\right) C_{x}^{2}-2 \psi_{i}^{2} \rho_{y x} C_{y} C_{x}\right],
$$

and:

$$
A_{2 i}=1+\theta\left[C_{y}^{2}+\left(2 \psi_{i}^{4}+\psi_{i}^{3}\right) C_{x}^{2}-4 \psi_{i}^{2} \rho_{y x} C_{y} C_{x}\right] .
$$

Putting the optimal values of $k$ in Relation (31), we have MSE as:

$$
\begin{aligned}
\operatorname{MSE}\left(\hat{\bar{Y}}_{P i}\right) \approx \bar{Y}^{2}\left[\left(\frac{A_{1 i}}{A_{2 i}}-1\right)^{2}+\left(\frac{A_{1 i}}{A_{2 i}}\right)^{2}\left(E\left(\xi_{0}^{2}\right)\right.\right. \\
\left.+\left(2 \psi_{i}^{4}+\psi_{i}^{3}\right) E\left(\xi_{1}^{2}\right)-4 \psi_{i}^{2} E\left(\xi_{0} \xi_{1}\right)\right) \\
\left.-\frac{A_{1 i}}{A_{2 i}}\left(\left(\psi_{i}^{4}+\psi_{i}^{3}\right) E\left(\xi_{1}^{2}\right)-2 \psi_{i}^{2} E\left(\xi_{0} \xi_{1}\right)\right)\right] .
\end{aligned}
$$

Simplifying Relation (32), we have the minimum MSE of the proposed ratio-type estimators:

$$
\operatorname{MSE}_{\min }\left(\hat{\bar{Y}}_{P i}\right) \approx \bar{Y}^{2}\left[1-\frac{A_{1 i}^{2}}{2 A_{2 i}}\right] .
$$


2.2. Second proposed family of ratio-type estimators

$$
\begin{aligned}
& \hat{\bar{Y}}_{P 2}=t\left[\bar{y}\left(\frac{\bar{X} \rho_{y x}+R}{\bar{x} \rho_{y x}+R}\right)^{\frac{\bar{X}_{\rho y x}}{\bar{X}_{\rho_{y x}+R}}}+b(\bar{X}-\bar{x})\right], \\
& \hat{\bar{Y}}_{P 4}=t\left[\bar{y}\left(\frac{\bar{X} \rho_{y x}+R C_{x}}{\bar{x} \rho_{y x}+R C_{x}}\right)^{\frac{\bar{x} \rho_{\rho_{y x}}}{\bar{x}_{\rho_{y x}+R C_{x}}}}+b(\bar{X}-\bar{x})\right], \\
& \hat{\bar{Y}}_{P 6}=t\left[\bar{y}\left(\frac{\bar{X} \rho_{y x}+R \beta_{2}(x)}{\bar{x} \rho_{y x}+R \beta_{2}(x)}\right)^{\frac{\bar{x}_{\rho x}}{\bar{X}_{y x}+R \beta_{2}(x)}}\right. \\
& +b(\bar{X}-\bar{x})], \\
& \hat{\bar{Y}}_{P 8}=t\left[\bar{y}\left(\frac{\bar{X} \rho_{y x}+R C_{x} \beta_{2}(x)}{\bar{x} \rho_{y x}+R C_{x} \beta_{2}(x)}\right)^{\frac{\bar{x}_{\rho_{y x}}}{\bar{x}_{\rho_{y x}+R C_{x} \beta_{2}(x)}}}\right. \\
& +b(\bar{X}-\bar{x})], \\
& \hat{\bar{Y}}_{P 10}=t\left[\bar{y}\left(\frac{\bar{X}+R \rho_{y x}}{\bar{x}+R \rho_{y x}}\right)^{\frac{\bar{x}+R \rho_{y x}}{\bar{x}}}+b(\bar{X}-\bar{x})\right], \\
& \hat{\bar{Y}}_{P 12}=t\left[\bar{y}\left(\frac{\bar{X}+R \rho_{y x} C_{x}}{\bar{x}+R \rho_{y x} C_{x}}\right)^{\frac{\bar{x}}{\bar{X}+R \rho_{y x} C_{x}}}+b(\bar{X}-\bar{x})\right] \text {, } \\
& \hat{\bar{Y}}_{P 14}=t\left[\bar{y}\left(\frac{\bar{X}+R \rho_{y x} \beta_{2}(x)}{\bar{x}+R \rho_{y x} \beta_{2}(x)}\right)^{\frac{\bar{x}+R \rho_{y x} \beta_{2}(x)}{\bar{x}}}\right. \\
& +b(\bar{X}-\bar{x})], \\
& \hat{\bar{Y}}_{P 16}=t\left[\bar{y}\left(\frac{\bar{X}+R \rho_{y x} C_{x} \beta_{2}(x)}{\bar{x}+R \rho_{y x} C_{x} \beta_{2}(x)}\right)^{\frac{\bar{x}}{\bar{x}+R \rho_{y x} C_{x} \beta_{2}(x)}}\right. \\
& +b(\bar{X}-\bar{x})],
\end{aligned}
$$

where $t$ is the unknown constant, which is to be determined later on, and $\bar{X}, C_{x}, \rho_{y x}$, and $\beta_{2}(x)$ are the known population parameters.

\section{Theorem 2.2}

The properties of $\hat{\bar{Y}}_{P j}$, i.e., $j=2,4,6, \ldots, 16$, are:

1) $\operatorname{Bias}\left(\hat{\bar{Y}}_{P j}\right) \approx \bar{Y}(t-1)$

$$
+\theta \bar{Y} t\left[\frac{1}{2}\left(\varphi_{j}^{4}+\varphi_{j}^{3}\right) C_{x}^{2}-\varphi_{j}^{2} \rho_{y x} C_{y} C_{x}\right]
$$

2) $\operatorname{MSE}\left(\hat{\bar{Y}}_{P j}\right) \approx\left[\left(\frac{A_{3 j}}{A_{4 j}} \bar{Y}-\bar{Y}\right)^{2}\right.$

$$
\begin{aligned}
& +\left(\frac{A_{3 j}}{A_{4 j}}\right)^{2}\left(\bar{Y}^{2} E\left(\xi_{1}^{2}\right)+\left\{2 \varphi_{j}^{4} \bar{Y}^{2}+b^{2} \bar{X}^{2}\right.\right. \\
& \left.+\varphi_{j}^{3} \bar{Y}^{2}+2 \varphi_{j}^{2} b \bar{Y} \bar{X}\right\} E\left(\xi_{1}^{2}\right)-\left\{4 \varphi_{j}^{2} \bar{Y}^{2}\right. \\
& \left.+2 b \bar{Y} \bar{X}\} E\left(\xi_{0} \xi_{1}\right)\right)-\frac{A_{3 j}}{A_{4 j}}\left(\left\{\varphi_{j}^{4} \bar{Y}^{2}\right.\right. \\
& \left.\left.\left.+\varphi_{j}^{3} \bar{Y}^{2}\right\} E\left(\xi_{1}^{2}\right)-2 \varphi_{j}^{2} \bar{Y}^{2} E\left(\xi_{0} \xi_{1}\right)\right)\right] .
\end{aligned}
$$

3) $\operatorname{MSE}_{\min }\left(\hat{\bar{Y}}_{P j}\right) \approx\left[\bar{Y}^{2}-\frac{A_{3 j}^{2}}{2 A_{4 j}}\right]$,

where:

$$
\begin{aligned}
& \varphi_{2}=\frac{\bar{X} \rho_{y x}}{\bar{X} \rho_{y x}+R}, \quad \varphi_{4}=\frac{\bar{X} \rho_{y x}}{\bar{X} \rho_{y x}+R C_{x}}, \\
& \varphi_{6}=\frac{\bar{X} \rho_{y x}}{\bar{X} \rho_{y x}+R \beta_{2}(x)}, \quad \varphi_{8}=\frac{\bar{X} \rho_{y x}}{\bar{X} \rho_{y x}+R C_{x} \beta_{2}(x)}, \\
& \varphi_{10}=\frac{\bar{X}}{\bar{X}+R \rho_{y x}}, \quad \varphi_{12}=\frac{\bar{X}}{\bar{X}+R \rho_{y x} C_{x}}, \\
& \varphi_{14}=\frac{\bar{X}}{\bar{X}+R \rho_{y x} \beta_{2}(x)}, \quad \varphi_{16}=\frac{\bar{X}}{\bar{X}+R \rho_{y x} C_{x} \beta_{2}(x)} .
\end{aligned}
$$

\section{Proof}

To prove Relations (42) to (44), the proposed estimators ( $\hat{\bar{Y}}_{P j}$, i.e., $\left.j=2,4,6, \ldots, 16\right)$ may be written in terms of $\xi_{i}^{\prime}$ s as follows:

$$
\hat{\bar{Y}}_{P j}=t\left[\left(\bar{Y}+\bar{Y} \xi_{0}\right)\left(1+\varphi_{j} \xi_{1}\right)^{-\varphi_{j}}-b \bar{X} \xi_{1}\right],
$$

or:

$$
\begin{aligned}
\hat{\bar{Y}}_{P j}= & t\left[\left(\bar{Y}+\bar{Y} \xi_{0}\right)\left\{1-\varphi_{j}^{2} \xi_{1}+\frac{1}{2} \varphi_{j}^{3}\left(\varphi_{j}+1\right) \xi_{1}^{2}\right\}\right. \\
& \left.-b \bar{X} \xi_{1}\right]
\end{aligned}
$$

where:

$$
b=\frac{\rho_{y x} S_{y}}{S_{x}} .
$$

Subtracting $\bar{Y}$ from Eq. (45) and expanding it up to the first degree of approximation, we obtain:

$$
\begin{aligned}
\hat{\bar{Y}}_{P j}-\bar{Y}= & {\left[t \bar{Y}+t \bar{Y} \xi_{0}-t \bar{Y} \varphi_{j}^{2} \xi_{1}-t b \bar{X} \xi_{1}\right.} \\
& \left.+\frac{1}{2} t \bar{Y}\left(\varphi_{j}^{4}+\varphi_{j}^{3}\right) \xi_{1}^{2}-t \bar{Y} \varphi_{j}^{2} \xi_{0} \xi_{1}-\bar{Y}\right] .
\end{aligned}
$$


The bias and the MSE of $\hat{\bar{Y}}_{P j}$, i.e., $j=2,4,6, \ldots, 16$, up to the first degree of approximation are given as:

$$
\begin{aligned}
& \operatorname{Bias}\left(\hat{\bar{Y}}_{P j}\right) \approx \bar{Y}(t-1)+\theta \bar{Y} t\left[\frac{1}{2}\left(\varphi_{j}^{4}+\varphi_{j}^{3}\right) C_{x}^{2}\right. \\
& \left.-\varphi_{j}^{2} \rho_{y x} C_{y} C_{x}\right] \\
& \operatorname{MSE}\left(\hat{\bar{Y}}_{P j}\right) \approx\left[(t \bar{Y}-\bar{Y})^{2}+t^{2}\left(\bar{Y}^{2} E\left(\xi_{0}^{2}\right)\right.\right. \\
& +\left\{2 \varphi_{j}^{4} \bar{Y}^{2}+b^{2} \bar{X}^{2}+\varphi_{j}^{3} \bar{Y}^{2}+2 \varphi_{j}^{2} b \bar{Y} \bar{X}\right\} E\left(\xi_{1}^{2}\right) \\
& \left.\quad-\left\{4 \varphi_{j}^{2} \bar{Y}^{2}+2 b \bar{Y} \bar{X}\right\} E\left(\xi_{0} \xi_{1}\right)\right) \\
& \left.-t\left(\left\{\varphi_{j}^{4} \bar{Y}^{2}+\varphi_{j}^{3} \bar{Y}^{2}\right\} E\left(\xi_{1}^{2}\right)-2 \varphi_{j}^{2} \bar{Y}^{2} E\left(\xi_{0} \xi_{1}\right)\right)\right] .
\end{aligned}
$$

Differentiating Eq. (48) with respect to $t$ and equating to zero, we have $t$ as shown in Box I, where:

$$
A_{3 j}=\bar{Y}^{2}+\frac{\theta}{2}\left[\left(\varphi_{j}^{4}+\varphi_{j}^{3}\right) R^{2} S_{x}^{2}-2 \varphi_{j}^{2} R S_{y x}\right],
$$

and:

$$
\begin{aligned}
A_{4 j}= & \bar{Y}^{2}+\theta\left[S_{y}^{2}+\left\{2 \varphi_{j}^{4} R^{2}+b^{2}+\varphi_{j}^{3} R^{2}\right.\right. \\
& \left.\left.+2 \varphi_{j}^{2} b R\right\} S_{x}^{2}-\left\{4 \varphi_{j}^{2} R+2 b\right\} S_{y x}\right] .
\end{aligned}
$$

Inserting the value of $t$ in Eq. (48), we obtain:

$$
\begin{aligned}
\operatorname{MSE}\left(\hat{\bar{Y}}_{P j}\right) \approx\left[\left(\frac{A_{3 j}}{A_{4 j}} \bar{Y}-\bar{Y}\right)^{2}+\left(\frac{A_{3 j}}{A_{4 j}}\right)^{2}\left(\bar{Y}^{2} E\left(\xi_{0}^{2}\right)\right.\right. \\
+\left\{2 \varphi_{j}^{4} \bar{Y}^{2}+b^{2} \bar{X}^{2}+\varphi_{j}^{3} \bar{Y}^{2}+2 \varphi_{j}^{2} b \bar{Y} \bar{X}\right\} E\left(\xi_{1}^{2}\right) \\
\left.\quad-\left\{4 \varphi_{j}^{2} \bar{Y}^{2}+2 b \bar{Y} \bar{X}\right\} E\left(\xi_{0} \xi_{1}\right)\right)
\end{aligned}
$$

$$
\left.-\frac{A_{3 j}}{A_{4 j}}\left(\left\{\varphi_{j}^{4} \bar{Y}^{2}+\varphi_{j}^{3} \bar{Y}^{2}\right\} E\left(\xi_{1}^{2}\right)-2 \varphi_{j}^{2} \bar{Y}^{2} E\left(\xi_{0} \xi_{1}\right)\right)\right]
$$

Thus, the minimum MSE is as follows:

$$
\operatorname{MSE}_{\min }\left(\hat{\bar{Y}}_{P j}\right) \approx\left[\bar{Y}^{2}-\frac{A_{3 j}^{2}}{2 A_{4 j}}\right] .
$$

\section{Interesting note}

Many more ratio-type estimators based on correlation coefficient can be formulated by taking different measures of $\psi_{i}$ and $\varphi_{j}$.

\section{Efficiency of the proposed estimators}

This section deals with the derivation of algebraic situations under which the proposed estimators will have minimum MSE as compared to unbiased estimator (sample mean), traditional ratio estimator, Singh and Tailor's [4] estimator, and Kadilar and Cingi's [24,25] classes of estimators.

\section{Theorem 3.1}

1. $\hat{\bar{Y}}_{P i}$ (i.e., $i=1,3,5, \ldots, 11$ ) perform better than $\hat{\bar{Y}}$ if:

$$
\left[\theta C_{y}^{2}+\frac{A_{1 i}^{2}}{2 A_{2 i}}\right]>1
$$

2. $\hat{\bar{Y}}_{P j}$ (i.e., $j=2,4,6, \ldots, 16$ ) perform better than $\hat{\bar{Y}}$ if:

$$
\left[\theta C_{y}^{2}+\frac{A_{3 j}^{2}}{2 \bar{Y}^{2} A_{4 j}}\right]>1
$$

\section{Proof}

1. By comparing Relations (2) and (33):

$$
\begin{aligned}
& \operatorname{MSE}_{\min }\left(\hat{\bar{Y}}_{P i}\right)<\operatorname{MSE}(\hat{\bar{Y}}), \\
& \bar{Y}^{2}\left[1-\frac{A_{1 i}^{2}}{2 A_{2 i}}\right]<\theta S_{y}^{2},
\end{aligned}
$$

$$
\begin{aligned}
& t=\frac{2 \bar{Y}^{2}+\left\{\varphi_{j}^{4} \bar{Y}^{2}+\varphi_{j}^{3} \bar{Y}^{2}\right\} E\left(\xi_{1}^{2}\right)-2 \varphi_{j}^{2} \bar{Y}^{2} E\left(\xi_{0} \xi_{1}\right)}{2 \bar{Y}^{2}+2\left[\bar{Y}^{2} E\left(\xi_{0}^{2}\right)+\left\{2 \varphi_{j}^{4} \bar{Y}^{2}+b^{2} \bar{X}^{2}+\varphi_{j}^{3} \bar{Y}^{2}+2 \varphi_{j}^{2} b \bar{Y} \bar{X}\right\} E\left(\xi_{1}^{2}\right)-\left\{4 \varphi_{j}^{2} \bar{Y}^{2}+2 b \bar{Y} \bar{X}\right\} E\left(\xi_{0} \xi_{1}\right)\right]}, \\
& t=\frac{\bar{Y}^{2}+\frac{\theta}{2}\left[\left(\varphi_{j}^{4}+\varphi_{j}^{3}\right) R^{2} S_{x}^{2}-2 \varphi_{j}^{2} R S_{y x}\right]}{\bar{Y}^{2}+\theta\left[S_{y}^{2}+\left\{2 \varphi_{j}^{4} R^{2}+b^{2}+\varphi_{j}^{3} R^{2}+2 \varphi_{j}^{2} b R\right\} S_{x}^{2}-\left\{4 \varphi_{j}^{2} R+2 b\right\} S_{y x}\right]}, \\
& t=\frac{A_{3 j}}{A_{4 j}}
\end{aligned}
$$




$$
\left[\theta C_{y}^{2}+\frac{A_{1 i}^{2}}{2 A_{2 i}}\right]>1 .
$$

2. By comparing Relations (2) and (50):

$$
\begin{aligned}
& \operatorname{MSE}_{\min }\left(\hat{\bar{Y}}_{P j}\right)<\operatorname{MSE}(\hat{\bar{Y}}), \\
& {\left[\bar{Y}^{2}-\frac{A_{3 j}^{2}}{2 A_{4 j}}\right]<\theta S_{y}^{2},} \\
& {\left[\theta C_{y}^{2}+\frac{A_{3 j}^{2}}{2 \bar{Y}^{2} A_{4 j}}\right]>1 .}
\end{aligned}
$$

\section{Theorem 3.2}

1. $\hat{\bar{Y}}_{P i}$ (i.e., $i=1,3,5, \ldots, 11$ ) perform better than traditional ratio estimator, $\hat{\bar{Y}}_{R}$, if:

$$
\theta\left[C_{y}^{2}+C_{x}^{2}(1-2 \varphi)\right]+\frac{A_{1 i}^{2}}{2 A_{2 i}}>1 .
$$

2. $\hat{\bar{Y}}_{P j}$ (i.e., $j=2,4,6, \ldots, 16$ ) perform better than traditional ratio estimator, $\hat{\bar{Y}}_{R}$, if:

$$
\theta\left[C_{y}^{2}+C_{x}^{2}(1-2 \varphi)\right]+\frac{A_{3 j}^{2}}{2 \bar{Y}^{2} A_{4 j}}>1 .
$$

\section{Proof}

1. By comparing Relations (5) and (33):

$$
\begin{aligned}
& \operatorname{MSE}_{\min }\left(\hat{\bar{Y}}_{P i}\right)<\operatorname{MSE}\left(\hat{\bar{Y}}_{R}\right), \\
& \bar{Y}^{2}\left[1-\frac{A_{1 i}^{2}}{2 A_{2 i}}\right]<\theta \bar{Y}^{2}\left[C_{y}^{2}+C_{x}^{2}(1-2 \varphi)\right], \\
& \theta\left[C_{y}^{2}+C_{x}^{2}(1-2 \varphi)\right]+\frac{A_{1 i}^{2}}{2 A_{2 i}}>1 .
\end{aligned}
$$

2. By comparing Relations (5) and (50):

$$
\begin{aligned}
& \operatorname{MSE}_{\text {min }}\left(\hat{\bar{Y}}_{P j}\right)<\operatorname{MSE}\left(\hat{\bar{Y}}_{R}\right), \\
& {\left[\bar{Y}^{2}-\frac{A_{3 j}^{2}}{2 A_{4 j}}\right]<\theta \bar{Y}^{2}\left[C_{y}^{2}+C_{x}^{2}(1-2 \varphi)\right],} \\
& \theta\left[C_{y}^{2}+C_{x}^{2}(1-2 \varphi)\right]+\frac{A_{3 j}^{2}}{2 \bar{Y}^{2} A_{4 j}}>1 .
\end{aligned}
$$

\section{Theorem 3.3}

1. $\hat{\bar{Y}}_{P i}$ (i.e., $i=1,3,5, \ldots, 11$ ) perform better than Singh and Tailor's [4] estimator, $\hat{\bar{Y}}_{S T}$, if:

$$
\theta\left[C_{y}^{2}+C_{x}^{2} \lambda(\lambda-2 \varphi)\right]+\frac{A_{1 i}^{2}}{2 A_{2 i}}>1 .
$$

2. $\hat{\bar{Y}}_{P j}$ (i.e., $j=2,4,6, \ldots, 16$ ) perform better than Singh and Tailor's [4] estimator, $\hat{\bar{Y}}_{S T}$, if:

$$
\theta\left[C_{y}^{2}+C_{x}^{2} \lambda(\lambda-2 \varphi)\right]+\frac{A_{3 j}^{2}}{2 \bar{Y}^{2} A_{4 j}}>1
$$

\section{Proof}

1. By comparing Relations (8) and (33):

$$
\begin{aligned}
& \operatorname{MSE}_{\min }\left(\hat{\bar{Y}}_{P i}\right)<\operatorname{MSE}\left(\hat{\bar{Y}}_{S T}\right), \\
& \bar{Y}^{2}\left[1-\frac{A_{1 i}^{2}}{2 A_{2 i}}\right]<\theta \bar{Y}^{2}\left[C_{y}^{2}+C_{x}^{2} \lambda(\lambda-2 \varphi)\right], \\
& \theta\left[C_{y}^{2}+C_{x}^{2} \lambda(\lambda-2 \varphi)\right]+\frac{A_{1 i}^{2}}{2 A_{2 i}}>1 .
\end{aligned}
$$

2. By comparing Relations (8) and (50):

$$
\begin{aligned}
& \operatorname{MSE}_{\min }\left(\hat{\bar{Y}}_{P j}\right)<\operatorname{MSE}\left(\hat{\bar{Y}}_{S T}\right), \\
& {\left[\bar{Y}^{2}-\frac{A_{3 j}^{2}}{2 A_{4 j}}\right]<\theta \bar{Y}^{2}\left[C_{y}^{2}+C_{x}^{2} \lambda(\lambda-2 \varphi)\right],} \\
& \theta\left[C_{y}^{2}+C_{x}^{2} \lambda(\lambda-2 \varphi)\right]+\frac{A_{3 j}^{2}}{2 \bar{Y}^{2} A_{4 j}}>1 .
\end{aligned}
$$

\section{Theorem 3.4}

1. $\hat{\bar{Y}}_{P i}$ (i.e., $i=1,3,5, \ldots, 11$ ) perform better than Kadilar and Cingi's [24] estimators, $\hat{\bar{Y}}_{K C i}$, if:

$$
\theta\left[C_{y}^{2}+C_{x}^{2} \lambda_{i}\left(\lambda_{i}-2 \varphi\right)\right]+\frac{A_{1 i}^{2}}{2 A_{2 i}}>1
$$

2. $\hat{\bar{Y}}_{P j}$ (i.e., $j=2,4,6, \ldots, 16$ ) perform better than Kadilar and Cingi's [24] estimators, $\hat{\bar{Y}}_{K C i}$, if:

$$
\theta\left[C_{y}^{2}+C_{x}^{2} \lambda_{i}\left(\lambda_{i}-2 \varphi\right)\right]+\frac{A_{3 j}^{2}}{2 \bar{Y}^{2} A_{4 j}}>1
$$

\section{Proof}

1. By comparing Relations (14) and (33):

$$
\begin{aligned}
& \operatorname{MSE}_{\min }\left(\hat{\bar{Y}}_{P i}\right)<\operatorname{MSE}\left(\hat{\bar{Y}}_{K C i}\right) \\
& \bar{Y}^{2}\left[1-\frac{A_{1 i}^{2}}{2 A_{2 i}}\right]<\theta \bar{Y}^{2}\left[C_{y}^{2}+C_{x}^{2} \lambda_{i}\left(\lambda_{i}-2 \varphi\right)\right] \\
& \theta\left[C_{y}^{2}+C_{x}^{2} \lambda_{i}\left(\lambda_{i}-2 \varphi\right)\right]+\frac{A_{1 i}^{2}}{2 A_{2 i}}>1
\end{aligned}
$$

2. By comparing Relations (14) and (50):

$$
\begin{aligned}
& \operatorname{MSE}_{\min }\left(\hat{\bar{Y}}_{P j}\right)<\operatorname{MSE}\left(\hat{\bar{Y}}_{K C i}\right), \\
& {\left[\bar{Y}^{2}-\frac{A_{3 j}^{2}}{2 A_{4 j}}\right]<\theta \bar{Y}^{2}\left[C_{y}^{2}+C_{x}^{2} \lambda_{i}\left(\lambda_{i}-2 \varphi\right)\right],} \\
& \theta\left[C_{y}^{2}+C_{x}^{2} \lambda_{i}\left(\lambda_{i}-2 \varphi\right)\right]+\frac{A_{3 j}^{2}}{2 \bar{Y}^{2} A_{4 j}}>1 .
\end{aligned}
$$




\section{Theorem 3.5}

1. $\hat{\bar{Y}}_{P i}$ (i.e., $\left.i=1,3,5, \ldots, 11\right)$ perform better than Kadilar and Cingi's [25] estimators, $\hat{\bar{Y}}_{K C i}^{*}$, if:

$$
\theta\left[\alpha_{i}^{* 2} C_{x}^{2}+C_{y}^{2}\left(1-\rho_{y x}^{2}\right)\right]+\frac{A_{1 i}^{2}}{2 A_{2 i}}>1 .
$$

2. $\hat{\bar{Y}}_{P j}$ (i.e., $\left.j=2,4,6, \ldots, 16\right)$ perform better than Kadilar and Cingi's [25] estimators, $\hat{\bar{Y}}_{K C i}^{*}$, if:

$$
\theta\left[\alpha_{i}^{* 2} C_{x}^{2}+C_{y}^{2}\left(1-\rho_{y x}^{2}\right)\right]+\frac{A_{3 j}^{2}}{2 \bar{Y}^{2} A_{4 j}}>1 .
$$

\section{Proof}

1. By comparing Relations (17) and (33):

$$
\begin{aligned}
& \operatorname{MSE}_{\text {min }}\left(\hat{\bar{Y}}_{P i}\right)<\operatorname{MSE}\left(\hat{\bar{Y}}_{K C i}^{*}\right), \\
& \bar{Y}^{2}\left[1-\frac{A_{1 i}^{2}}{2 A_{2 i}}\right]<\theta \bar{Y}^{2}\left[\alpha_{i}^{* 2} C_{x}^{2}+C_{y}^{2}\left(1-\rho_{y x}^{2}\right)\right], \\
& \theta\left[\alpha_{i}^{* 2} C_{x}^{2}+C_{y}^{2}\left(1-\rho_{y x}^{2}\right)\right]+\frac{A_{1 i}^{2}}{2 A_{2 i}}>1 .
\end{aligned}
$$

2. By comparing Relations (17) and (50):

$$
\begin{aligned}
& \operatorname{MSE}_{\min }\left(\hat{\bar{Y}}_{P j}\right)<\operatorname{MSE}\left(\hat{\bar{Y}}_{K C i}^{*}\right), \\
& {\left[\bar{Y}^{2}-\frac{A_{3 j}^{2}}{2 A_{4 j}}\right]<\theta \bar{Y}^{2}\left[\alpha_{i}^{* 2} C_{x}^{2}+C_{y}^{2}\left(1-\rho_{y x}^{2}\right)\right],} \\
& \theta\left[\alpha_{i}^{* 2} C_{x}^{2}+C_{y}^{2}\left(1-\rho_{y x}^{2}\right)\right]+\frac{A_{3 j}^{2}}{2 \bar{Y}^{2} A_{4 j}}>1 .
\end{aligned}
$$

\section{Empirical study}

This section evaluates the performance of the proposed families of ratio-type estimators, i.e., ( $\hat{\bar{Y}}_{P i}$ i.e., $i=$ $1,3,5, \ldots, 11)$ and $\left(\hat{\bar{Y}}_{P j}\right.$ i.e., $\left.j=2,4,6, \ldots, 16\right)$, with competitive estimators. For this purpose, we consider two natural populations described below.

\section{Population 1 (source: Kadilar and Cingi [24]):}

The data relates to 104 villages of East Anatolia Region of Turkey in 1999. The following variables are taken into consideration:

$y \quad$ The level of apple production (in 1000 tones);

$x \quad$ The number of apple trees.

Values of different required parameters are: $N=104$, $n=20, \rho_{y x}=0.865, \bar{Y}=625.37, \bar{X}=13.931, C_{y}=$ $1.866, C_{x}=1.653$, and $\beta_{2}(x)=17.52$.

\section{Population 2 (source: Murthy [26]):}

The variables are defined as follows:

$y \quad$ The output for 80 factories;

$x \quad$ The number of workers.

Values of different required parameters are: $N=80$, $n=20, \rho_{y x}=0.9150, \bar{Y}=51.8264, \bar{X}=2.8513$, $C_{y}=0.3542, C_{x}=0.9484, S_{y}=18.3569, S_{x}=2.7042$, and $\beta_{2}(x)=1.3005$.

Consider the MSE given in Section 1 and derived in Section 2; the values for existing and proposed families of estimators are computed and placed in Tables 1 and 2. The following are some important findings observed in Tables 1 and 2:

- It is important to note that the traditional ratio estimator is more efficient than the suggested class of estimators by Kadilar and Cingi [25], i.e., $\hat{\bar{Y}}_{K C 1}^{*}$, $\hat{\bar{Y}}_{K C 2}^{*}, \hat{\bar{Y}}_{K C 3}^{*}, \hat{\bar{Y}}_{K C 4}^{*}$, and $\hat{\bar{Y}}_{K C 5}^{*}$, for both real populations discussed in this manuscript;

- It can be concluded that proposed families of ratiotype estimators are more efficient as they have lesser values of MSE as compared to the usual unbiased estimator, traditional ratio estimator, Singh and Tailor's [4] estimator, and Kadilar and Cingi's [24,25] classes of estimators;

- It is perceived in both populations that the two proposed estimators $\hat{\bar{Y}}_{P 11}$ and $\hat{\bar{Y}}_{P 6}$ have the least

Table 1. MSEs of the competing estimators for Population 1.

\begin{tabular}{cccccccc}
\hline Estimators & MSE & Estimators & MSE & Estimators & MSE & Estimators & MSE \\
\hline$\hat{\bar{Y}}$ & 54993.750 & $\hat{\bar{Y}}_{K C 1}^{*}$ & 52102.800 & $\hat{\bar{Y}}_{P 5}$ & 13335.710 & $\hat{\bar{Y}}_{P 8}$ & 13372.840 \\
$\hat{\bar{Y}}_{R}$ & 13869.960 & $\hat{\bar{Y}}_{K C 2}^{*}$ & 53933.140 & $\hat{\bar{Y}}_{P 7}$ & 13341.230 & $\hat{\bar{Y}}_{P 10}$ & 13718.360 \\
$\hat{\bar{Y}}_{S T}$ & 13898.700 & $\hat{\bar{Y}}_{K C 3}^{*}$ & 47217.400 & $\hat{\bar{Y}}_{P 9}$ & 13847.380 & $\hat{\bar{Y}}_{P 12}$ & 13489.420 \\
$\hat{\bar{Y}}_{K C 1}$ & 13852.970 & $\hat{\bar{Y}}_{K C 4}^{*}$ & 56697.220 & $\hat{\bar{Y}}_{P 11}$ & $\mathbf{1 3 3 2 8 . 1 5 0}$ & $\hat{\bar{Y}}_{P 14}$ & 13373.720 \\
$\hat{\bar{Y}}_{K C 2}$ & 14252.910 & $\hat{\bar{Y}}_{K C 5}^{*}$ & 21012.160 & $\hat{\bar{Y}}_{P 2}$ & 13556.390 & $\hat{\bar{Y}}_{P 16}$ & 13373.020 \\
$\hat{\bar{Y}}_{K C 3}$ & 13863.330 & $\hat{\bar{Y}}_{P 1}$ & 13540.760 & $\hat{\bar{Y}}_{P 4}$ & 13436.650 & & \\
$\hat{\bar{Y}}_{K C 4}$ & 27816.360 & $\hat{\bar{Y}}_{P 3}$ & 13330.560 & $\hat{\bar{Y}}_{P 6}$ & 13373.240 & & \\
\hline
\end{tabular}

*Bold value indicates minimum MSE. 
Table 2. MSEs of the competing estimators for Population 2.

\begin{tabular}{cccccccc}
\hline Estimators & MSE & Estimators & MSE & Estimators & MSE & Estimators & MSE \\
\hline$\hat{\hat{Y}}$ & 12.6366 & $\hat{\bar{Y}}_{K C 1}^{*}$ & 53.9814 & $\hat{\bar{Y}}_{P 5}$ & 10.6136 & $\hat{\bar{Y}}_{P 8}$ & 2.0664 \\
$\hat{\bar{Y}}_{R}$ & 41.3150 & $\hat{\bar{Y}}_{K C 2}^{*}$ & 52.6355 & $\hat{\bar{Y}}_{P 7}$ & 8.3250 & $\hat{\bar{Y}}_{P 10}$ & 2.0975 \\
$\hat{\bar{Y}}_{S T}$ & 17.6849 & $\hat{\bar{Y}}_{K C 3}^{*}$ & 50.7866 & $\hat{\bar{Y}}_{P 9}$ & 6.7023 & $\hat{\bar{Y}}_{P 12}$ & 2.1057 \\
$\hat{\bar{Y}}_{K C 1}$ & 16.9505 & $\hat{\bar{Y}}_{K C 4}^{*}$ & 60.3414 & $\hat{\bar{Y}}_{P 11}$ & 9.2264 & $\hat{\bar{Y}}_{P 14}$ & 2.0724 \\
$\hat{\bar{Y}}_{K C 2}$ & 15.9550 & $\hat{\bar{Y}}_{K C 5}^{*}$ & 42.4043 & $\hat{\bar{Y}}_{P 2}$ & 2.0783 & $\hat{\bar{Y}}_{P 16}$ & 2.0759 \\
$\hat{\bar{Y}}_{K C 3}$ & 21.2570 & $\hat{\bar{Y}}_{P 1}$ & 6.1271 & $\hat{\bar{Y}}_{P 4}$ & 2.0829 & & \\
$\hat{\bar{Y}}_{K C 4}$ & 11.6626 & $\hat{\bar{Y}}_{P 3}$ & 9.9128 & $\hat{\bar{Y}}_{P 6}$ & $\mathbf{2 . 0 6 4 5}$ & & \\
\hline
\end{tabular}

*Bold value indicates minimum MSE.

Table 3. PREs of the competing estimators for Population 1.

\begin{tabular}{|c|c|c|c|c|c|c|c|c|c|c|c|c|}
\hline \multirow{2}{*}{$\begin{array}{l}\text { Proposed } \\
\text { estimators }\end{array}$} & \multicolumn{12}{|c|}{ Existing estimators } \\
\hline & $\hat{\overline{\mathbf{Y}}}$ & $\hat{\bar{Y}}_{R}$ & $\hat{\bar{Y}}_{S T}$ & $\hat{\bar{Y}}_{K C 1}$ & $\hat{\bar{Y}}_{K C 2}$ & $\hat{\bar{Y}}_{K C 3}$ & $\hat{\bar{Y}}_{K C 4}$ & $\hat{\bar{Y}}_{K C 1}^{*}$ & $\hat{\bar{Y}}_{K C 2}^{*}$ & $\hat{\bar{Y}}_{K C 3}^{*}$ & $\hat{\bar{Y}}_{K C 4}^{*}$ & $\hat{\bar{Y}}_{K C 5}^{*}$ \\
\hline$\hat{\bar{Y}}_{P 1}$ & 406.1349 & 102.4312 & 102.6434 & 102.3057 & 105.2593 & 102.3822 & 205.4269 & 384.7849 & 398.3022 & 348.7057 & 418.7152 & 155.1771 \\
\hline$\hat{\bar{Y}}_{P 3}$ & 412.5389 & 104.0463 & 104.2619 & 103.9189 & 106.9191 & 103.9966 & 208.6661 & 390.8523 & 404.5827 & 354.2042 & 425.3176 & 157.6240 \\
\hline$\hat{\bar{Y}}_{P 5}$ & 412.3796 & 104.0062 & 104.2217 & 103.8788 & 106.8778 & 103.9564 & 208.5855 & 390.7014 & 404.4265 & 354.0674 & 425.1534 & 157.5631 \\
\hline$\hat{\bar{Y}}_{P 7}$ & 412.2090 & 103.9631 & 104.1786 & 103.8358 & 106.8336 & 103.9134 & 208.4992 & 390.5397 & 404.2591 & 353.9209 & 424.9775 & 157.4979 \\
\hline$\hat{\bar{Y}}_{P 9}$ & 397.1419 & 100.1631 & 100.3706 & 100.0404 & 102.9286 & 100.1152 & 200.8781 & 376.2647 & 389.4826 & 340.9844 & 409.4437 & 151.7411 \\
\hline$\hat{\bar{Y}}_{P 11}$ & 412.6135 & 104.0652 & 104.2808 & 103.9377 & 106.9384 & 104.0154 & 208.7038 & 390.9230 & 404.6559 & 354.2682 & 425.3945 & 157.6525 \\
\hline$\hat{\bar{Y}}_{P 2}$ & 405.6666 & 102.3131 & 102.5251 & 102.1878 & 105.1379 & 102.2642 & 205.1900 & 384.3413 & 397.8429 & 348.3036 & 418.2324 & 154.9982 \\
\hline$\hat{\bar{Y}}_{P 4}$ & 409.2817 & 103.2248 & 103.4387 & 103.0984 & 106.0749 & 103.1755 & 207.0186 & 387.7663 & 401.3883 & 351.4075 & 421.9595 & 156.3795 \\
\hline$\hat{\bar{Y}}_{P 6}$ & 411.2223 & 103.7143 & 103.9292 & 103.5872 & 106.5778 & 103.6647 & 208.0002 & 389.6049 & 403.2915 & 353.0738 & 423.9602 & 157.1209 \\
\hline$\hat{\bar{Y}}_{P 8}$ & 411.2346 & 103.7174 & 103.9323 & 103.5903 & 106.5810 & 103.6678 & 208.0064 & 389.6166 & 403.3036 & 353.0843 & 423.9729 & 157.1256 \\
\hline$\hat{\bar{Y}}_{P 10}$ & 400.8770 & 101.1051 & 101.3146 & 100.9812 & 103.8966 & 101.0568 & 202.7674 & 379.8034 & 393.1457 & 344.1913 & 413.2944 & 153.1682 \\
\hline$\hat{\bar{Y}}_{P 12}$ & 407.6806 & 102.8210 & 103.0341 & 102.6951 & 105.6599 & 102.7719 & 206.2087 & 386.2494 & 399.8181 & 350.0328 & 420.3088 & 155.7677 \\
\hline$\hat{\bar{Y}}_{P 14}$ & 411.2076 & 103.7106 & 103.9255 & 103.5835 & 106.5740 & 103.6610 & 207.9927 & 389.5909 & 403.2770 & 353.0611 & 423.9450 & 157.1153 \\
\hline$\hat{\bar{Y}}_{P 16}$ & 411.2291 & 103.7160 & 103.9309 & 103.5889 & 106.5796 & 103.6664 & 208.0036 & 389.6113 & 403.2981 & 353.0796 & 423.9672 & 157.1235 \\
\hline
\end{tabular}

MSE values (13328.150 and 2.0645) among all the proposed estimators;

- It is interesting to note that the Kadilar and Cingi's [24] estimators, $\hat{\bar{Y}}_{K C 1}$ and $\hat{\bar{Y}}_{K C 3}$, are the special cases of the proposed estimators, $\hat{\bar{Y}}_{P 1}$ and $\hat{\bar{Y}}_{P 3}$. When we put the values of $k=1$ and $\psi_{i}=1$, where $i=1$ and 2 , in $\hat{\bar{Y}}_{P 1}$ and $\hat{\bar{Y}}_{P 3}$, these estimators give the same result as the estimators $\hat{\bar{Y}}_{K C 1}$ and $\hat{\bar{Y}}_{K C 3}$.

This study calculated the Percentage Relative Efficiencies (PREs) for both populations under consideration to prove the dominance of the proposed families of ratio-type estimators, i.e., $\left(\hat{\bar{Y}}_{P i}\right.$, i.e., $i=$ $1,3,5, \ldots, 11)$ and $\left(\hat{\bar{Y}}_{P j}\right.$, i.e., $\left.j=2,4,6, \ldots, 16\right)$, over the existing estimators, i.e., $\hat{\bar{Y}}, \hat{\bar{Y}}_{R}, \hat{\bar{Y}}_{S T}, \hat{\bar{Y}}_{K C 1}, \hat{\bar{Y}}_{K C 2}$, $\hat{\bar{Y}}_{K C 3}, \hat{\bar{Y}}_{K C 4}, \hat{\bar{Y}}_{K C 1}^{*}, \hat{\bar{Y}}_{K C 2}^{*}, \hat{\bar{Y}}_{K C 3}^{*}, \hat{\bar{Y}}_{K C 4}^{*}$, and $\hat{\bar{Y}}_{K C 5}^{*}$. Here, the Percentage Relative Efficiency (PRE) is the ratio of MSE of the existing estimators $(e)$ to the MSE of the proposed ratio-type estimators $(p)$ and is given by:

$$
\operatorname{PRE}(e, p)=\frac{\operatorname{MSE}(e)}{\operatorname{MSE}(p)} \times 100 .
$$

Tables 3 and 4 provide the PREs calculated for both populations. It is revealed in Tables 3 and 4 that the proposed families of ratio-type estimators are more competent than the existing estimators for both populations as they have higher values.

To get deeper insight, another important tool, i.e., Relative Root Mean Square Error (RRMSE), is computed in this study. It is the most useful measure to compare the precision of the estimators (see, e.g., $[12,22,27,28]$ ). The RRMSE of an estimator can be calculated by the relation given below:

$$
\operatorname{RRMSE}=\frac{\sqrt{\operatorname{MSE}(\hat{\phi})}}{\phi},
$$

where MSE may be defined as:

$$
\operatorname{MSE}(\hat{\phi})=\frac{1}{n} \sum_{i=1}^{n}(\hat{\phi}-\phi)^{2}
$$

where $\hat{\phi}$ is the estimate of $\phi$ on the $i$ th sample.

It is quite obvious from the values of RRMSE shown in Tables 5 and 6 that the proposed families of ratio-type estimators perform better than the existing ones discussed in this article. 
Table 4. PREs of the competing estimators for Population 2.

\begin{tabular}{|c|c|c|c|c|c|c|c|c|c|c|c|c|}
\hline \multirow{2}{*}{$\begin{array}{c}\text { Proposed } \\
\text { estimators }\end{array}$} & \multicolumn{12}{|c|}{ Existing estimators } \\
\hline & $\hat{\overline{\boldsymbol{Y}}}$ & $\hat{\bar{Y}}_{R}$ & $\hat{\bar{Y}}_{S T}$ & $\hat{\bar{Y}}_{K C 1}$ & $\hat{\bar{Y}}_{K C 2}$ & $\hat{\bar{Y}}_{K C 3}$ & $\hat{\bar{Y}}_{K C 4}$ & $\hat{\bar{Y}}_{K C 1}^{*}$ & $\hat{\bar{Y}}_{K C 2}^{*}$ & $\hat{\bar{Y}}_{K C 3}^{*}$ & $\hat{\bar{Y}}_{K C 4}^{*}$ & $\hat{\overline{\boldsymbol{Y}}}_{K C 5}^{*}$ \\
\hline$\hat{\bar{Y}}_{P 1}$ & 206.2 & 74.2994 & 88.6341 & 276.6480 & 260.4005 & 346.9341 & 190.3445 & 881.0269 & 859.0606 & 828.8848 & 984.8281 & 692.0778 \\
\hline$\hat{\bar{Y}}_{P 3}$ & 127.4776 & 416.7844 & 178.4047 & 170.9961 & 160.9535 & 214.4399 & 117.6519 & 544.5626 & 530.9852 & 512.3335 & 608.7221 & 427.7732 \\
\hline$\hat{\bar{Y}}_{P 5}$ & 119.0605 & 389.2647 & 166.6249 & 159.7055 & 150.3260 & 200.2808 & 109.8835 & 508.6059 & 495.9250 & 478.5049 & 568.5291 & 399.5280 \\
\hline$\hat{\bar{Y}}_{P 7}$ & 151.7910 & 496.2763 & 212.4312 & 203.6096 & 191.6517 & 255.3393 & 140.0913 & 648.4252 & 632.2583 & 610.0492 & 724.8216 & 509.3610 \\
\hline$\hat{\bar{Y}}_{P 9}$ & 188.5412 & 616.4302 & 263.8632 & 252.9057 & 238.0526 & 317.1598 & 174.0089 & 805.4161 & 785.3349 & 757.7488 & 900.3088 & 632.6828 \\
\hline$\hat{\bar{Y}}_{P 11}$ & 136.9613 & 447.7911 & 191.6771 & 183.7174 & 172.9277 & 230.3932 & 126.4047 & 585.0754 & 570.4879 & 550.4487 & 654.0081 & 459.5975 \\
\hline$\hat{\bar{Y}}_{P 2}$ & 608.0258 & 1987.9228 & 850.9310 & 815.5945 & 767.6948 & 1022.8071 & 561.1606 & 2597.3825 & 2532.6228 & 2443.6607 & 2903.4018 & 2040.3359 \\
\hline$\hat{\bar{Y}}_{P 4}$ & 606.6830 & 1983.5326 & 849.0518 & 813.7933 & 765.9993 & 1020.5483 & 559.9213 & 2591.6463 & 2527.0296 & 2438.2640 & 2896.9898 & 2035.8299 \\
\hline$\hat{\bar{Y}}_{P 6}$ & 612.0901 & 2001.2109 & 856.6190 & 821.0463 & 772.8264 & 1029.6440 & 564.9116 & 2614.7445 & 2549.5519 & 2459.9952 & 2922.8094 & 2053.9743 \\
\hline$\hat{\bar{Y}}_{P 8}$ & 611.5273 & 1999.3709 & 855.8314 & 820.2913 & 772.1158 & 1028.6973 & 564.3922 & 2612.3403 & 2547.2077 & 2457.7333 & 2920 & 2052.0858 \\
\hline$\hat{\bar{Y}}_{P 10}$ & 602.4601 & 1969.7259 & 843.1418 & 808.1287 & 760.6675 & 1013.4446 & 556.0238 & 2573.6067 & 2509.4398 & 2421.2920 & 2876.8248 & 2021.6591 \\
\hline$\hat{\bar{Y}}_{P 12}$ & 600.1140 & 1962.0554 & 839.8585 & 804.9817 & 757.7053 & 1009.4980 & 553.8586 & 2563.5846 & 2499.6676 & 2411.8630 & 2865.6219 & 2013.7864 \\
\hline$\hat{\bar{Y}}_{P 14}$ & 609.7568 & 1993.5823 & 853.3536 & 817.9164 & 769.8803 & 1025.7190 & 562.7582 & 2604.7771 & 2539.8330 & 2450.6176 & 2911.6676 & 2046.1446 \\
\hline$\hat{\bar{Y}}_{P 16}$ & 608.7287 & 1990.2211 & 851.9148 & 816.5374 & 768.5823 & 1023.9896 & 561.8093 & 2600.3854 & 2535.5508 & 2446.4859 & 2906.7585 & 2042.6947 \\
\hline
\end{tabular}

Table 5. RRMSEs of the competing estimators for Population 1.

\begin{tabular}{cccccccc}
\hline Estimators & RRMSE & Estimators & RRMSE & Estimators & RRMSE & Estimators & RRMSE \\
\hline$\hat{\bar{Y}}$ & 0.3750 & $\hat{\bar{Y}}_{K C 1}^{*}$ & 0.3650 & $\hat{\bar{Y}}_{P 5}$ & 0.1846 & $\hat{\bar{Y}}_{P 8}$ & 0.1849 \\
$\hat{\bar{Y}}_{R}$ & 0.1883 & $\hat{\bar{Y}}_{K C 2}^{*}$ & 0.3714 & $\hat{\bar{Y}}_{P 7}$ & 0.1847 & $\hat{\bar{Y}}_{P 10}$ & 0.1872 \\
$\hat{\bar{Y}}_{S T}$ & 0.1885 & $\hat{\bar{Y}}_{K C 3}^{*}$ & 0.3475 & $\hat{\bar{Y}}_{P 9}$ & 0.1881 & $\hat{\bar{Y}}_{P 12}$ & 0.1857 \\
$\hat{\bar{Y}}_{K C 1}$ & 0.1882 & $\hat{\bar{Y}}_{K C 4}^{*}$ & 0.3808 & $\hat{\bar{Y}}_{P 11}$ & 0.1846 & $\hat{\bar{Y}}_{P 14}$ & 0.1849 \\
$\hat{\bar{Y}}_{K C 2}$ & 0.1909 & $\hat{\bar{Y}}_{K C 5}^{*}$ & 0.2318 & $\hat{\bar{Y}}_{P 2}$ & 0.1861 & $\hat{\bar{Y}}_{P 16}$ & 0.1849 \\
$\hat{\bar{Y}}_{K C 3}$ & 0.1883 & $\hat{\bar{Y}}_{P 1}$ & 0.1860 & $\hat{\bar{Y}}_{P 4}$ & 0.1853 & & \\
$\hat{\bar{Y}}_{K C 4}$ & 0.2667 & $\hat{\bar{Y}}_{P 3}$ & 0.1846 & $\hat{\bar{Y}}_{P 6}$ & 0.1849 & & \\
\hline
\end{tabular}

Table 6. RRMSEs of the competing estimators for Population 2.

\begin{tabular}{cccccccc}
\hline Estimators & RRMSE & Estimators & RRMSE & Estimators & RRMSE & Estimators & RRMSE \\
\hline$\hat{\bar{Y}}$ & 0.0686 & $\hat{\bar{Y}}_{K C 1}^{*}$ & 0.1418 & $\hat{\bar{Y}}_{P 5}$ & 0.0628 & $\hat{\bar{Y}}_{P 8}$ & 0.0277 \\
$\hat{\bar{Y}}_{R}$ & 0.1240 & $\hat{\bar{Y}}_{K C 2}^{*}$ & 0.1400 & $\hat{\bar{Y}}_{P 7}$ & 0.0556 & $\hat{\bar{Y}}_{P 10}$ & 0.0279 \\
$\hat{\bar{Y}}_{S T}$ & 0.0811 & $\hat{\bar{Y}}_{K C 3}^{*}$ & 0.1375 & $\hat{\bar{Y}}_{P 9}$ & 0.0499 & $\hat{\bar{Y}}_{P 12}$ & 0.0280 \\
$\hat{\bar{Y}}_{K C 1}$ & 0.0794 & $\hat{\bar{Y}}_{K C 4}^{*}$ & 0.1499 & $\hat{\bar{Y}}_{P 11}$ & 0.0586 & $\hat{\bar{Y}}_{P 14}$ & 0.0277 \\
$\hat{\hat{Y}}_{K C 2}$ & 0.0771 & $\hat{\bar{Y}}_{K C 5}^{*}$ & 0.1256 & $\hat{\bar{Y}}_{P 2}$ & 0.0278 & $\hat{\bar{Y}}_{P 16}$ & 0.0278 \\
$\hat{\bar{Y}}_{K C 3}$ & 0.0890 & $\hat{\bar{Y}}_{P 1}$ & 0.0477 & $\hat{\bar{Y}}_{P 4}$ & 0.0278 & & \\
$\hat{\bar{Y}}_{K C 4}$ & 0.0659 & $\hat{\bar{Y}}_{P 3}$ & 0.0607 & $\hat{\bar{Y}}_{P 6}$ & 0.0277 & & \\
\hline
\end{tabular}

\section{Conclusion}

In this article, we proposed improved families of ratiotype estimators of population mean under simple random sampling without replacement (SRSWOR) scheme using correlation coefficient between study and auxiliary variables. We obtained bias, Mean Square Error (MSE), and minimum MSE formulae of the proposed families of ratio-type estimators and compared them theoretically with those of the traditional and exiting modified ratio estimators in the literature. It was found that the newly proposed estimators were more efficient than the traditional estimators, such as usual unbiased and ratio, i.e., $\hat{\bar{Y}}$ and $\hat{\bar{Y}}_{R}$, and existing modified ratio estimators, i.e., $\hat{\bar{Y}}_{S T}$ (Singh and Tailor [4]), $\hat{\bar{Y}}_{K C i}$ (Kadilar and Cingi [24]), and $\hat{\bar{Y}}_{K C i}^{*}$
(Kadilar and Cingi [25]), in terms of Mean Squared Error (MSE), Percentage Relative Efficiency (PRE), and Relative Root Mean Square Error (RRMSE). It was also empirically observed that the proposed families of ratio-type estimators performed better than the traditional and existing modified ratio estimators by using two natural population data sets. Hence, we strongly suggest the use of our newly proposed ratiotype estimators over the existing ratio-type estimators used in this study for future work.

\section{Acknowledgments}

The authors are heartily thankful to the unknown learned referees for their valuable remarks and encouragements. 


\section{References}

1. Cochran, W.G. "The estimation of the yields of cereal experiments by sampling for the ratio gain to total produce", Journal of Agriculture Science, 30, pp. 262275 (1940).

2. Sisodia, B.V.S. and Dwivedi, V.K. "A modified ratio estimator using coefficient of variation of auxiliary variable", Journal of the Indian Society of Agriculture Statistics, 33(1), pp. 13-18 (1981).

3. Upadhyaya, L.N. and Singh, H.P. "Use of transformed auxiliary variable in estimating the finite population mean", Biometrical Journal, 41(5), pp. 627-636 (1999).

4. Singh, H.P. and Tailor, R. "Use of known correlation coefficient in estimating the finite population means", Statistics in Transition, 6(4), pp. 555-560 (2003).

5. Kadilar, C. and Cingi, H. "Ratio estimators in simple random sampling", Applied Mathematics and Computation, 151, pp. 893-902 (2004).

6. Kadilar, C. and Cingi, H. "Improvement in estimating the population mean in simple random sampling", Applied Mathematics Letters, 19, pp. 75-79 (2006c).

7. Singh, H.P., Tailor, R., Tailor, R., and Kakran, M.S. "An improved estimator of population mean using power transformation", Journal of the Indian Society of Agriculture Statistics, 58(2), pp. 223-230 (2004).

8. Singh, R., Chauhan, P., and Sawan, N. "A family of estimators for estimating population means using known correlation coefficient in two-phase sampling", Statistics in Transition, New Series, 8(1), pp. 89-96 (2007).

9. Gupta, S. and Shabbir, J. "On improvement in estimating the population mean in simple random sampling", Journal of Applied Statistics, 35(5), pp. 559566 (2008).

10. Koyuncu, N. and Kadilar, C. "Efficient estimators for the population mean", Hacettepe Journal of Mathematics and Statistics, 38(2), pp. 217-225 (2009).

11. Koyuncu, N. and Kadilar, C. "On improvement in estimating population mean in stratified random sampling", Journal of Applied Statistics, 37(6), pp. 9991013 (2010).

12. Yan, Z. and Tian, B. "Ratio method to the mean estimation using coefficient of skewness of auxiliary variable", ICICA. Part II, CCIS, 106, pp. 103-110 (2010).

13. Haq, A. and Shabbir, J. "Improved family of ratio estimators in simple and stratified random sampling", Communications in Statistics - Theory and Methods, 42, pp. 782-799 (2013).

14. Singh, H.P. and Solanki, R.S. "An efficient class of estimators for the population mean using auxiliary information", Communications in Statistics-Theory and Methods, 42, pp. 145-163 (2013).
15. Yadav, S.K. and Kadilar, C. "Improved class of ratio and product estimators", Applied Mathematics and Computation, 219, pp. 10726-10731 (2013).

16. Subramani, J. and Kumarapandiyan, G. "Modified ratio estimators using known median and coefficient of kurtosis", American Journal of Mathematics and Statistics, 2(4), pp. 95-100 (2012).

17. Subramani, J. and Kumarapandiyan, G. "Estimation of population mean using known correlation coefficient and median", Journal of Statistical Theory and Applications, 13(4), pp. 333-343 (2014).

18. Subramani, J. and Prabavathy, G. "Median based modified ratio estimators with known quartiles of an auxiliary variable", Journal of Modern Applied Statistical Methods, 13(1), pp. 234-254 (2014).

19. Yadav, S.K., Misra, S., Shukla, A.K., and Tiwari, V. "Improved estimation of population mean using median and coefficient of variation of auxiliary variable", International Journal of Engineering Research and Applications, 4(1), pp. 206-211 (2014).

20. Khan, S.A., Ali, H., Manzoor, S., and Alamgir. "A class of transformed efficient ratio estimators of finite population mean", Pakistan Journal of Statistics, 31(4), pp. 353-362 (2015).

21. Kumar, S. "An estimator of the mean estimation of study variable using median of auxiliary variable", Sri Lankan Journal of Applied Statistics, 16(2), pp. 107115 (2015).

22. Irfan, M., Javed, M., Abid, M., and Lin, Z. "Improved ratio type estimators of population mean based on median of a study variable and an auxiliary variable", Hacettepe Journal of Mathematics and Statistics, 47(3), pp. 659-673 (2018).

23. Walia, G.S., Kaur, H., and Sharma, M.K. "Ratio type estimator of population mean through efficient linear transformation", American Journal of Mathematics and Statistics, 5(3), pp. 144-149 (2015).

24. Kadilar, C. and Cingi, H. "An improvement in estimating the population mean by using the correlation coefficient", Hacettepe Journal of Mathematics and Statistics, 35(1), pp. 103-109 (2006a).

25. Kadilar, C. and Cingi, H. "New ratio estimators using correlation coefficient", Interstat., pp. 1-11 (2006b).

26. Murthy, M.N., Sampling Theory and Methods, Statistical Publishing Society, Calcutta, India (1967).

27. Munoz, J.F., Alvarez, E., and Rueda, M.M. "Optimum design-based ratio estimators of the distribution function", Journal of Applied Statistics, 41(7), pp. 13951407 (2014).

28. Alvarez, E., Moya-Fernandez, P.J., BlancoEncomienda, F.J., and Munoz, J. F. "Methodological insights for industrial quality control management: The impact of various estimators of the standard deviation on the process capability index", Journal of King Saud University Science, 27, pp. 271-277 (2015). 


\section{Biographies}

Muhammad Irfan is a doctoral student of Statistics at Zhejiang University, China. He has been working as lecturer in the Department of Statistics, Government College University, Faisalabad, Pakistan, since 2004. He has 11 research papers published in reputed journals. His areas of interest are survey sampling, probability distributions, and time series analysis.

Maria Javed is a doctoral student of Statistics at Zhejiang University, China. She has been working as lecturer in the Department of Statistics, Government College University, Faisalabad, Pakistan, since 2004. She has more than 8 research publications in international research journals. Her fields of work are probability distributions and survey sampling.

Zhengyan Lin is a Professor of Statistics at Zhejiang University, China. He has over 230 refereed publications and 8 edited book volumes, and has guided research students at all levels of the curriculum, including undergraduate and $\mathrm{PhD}$ students. He has received external funding from National Natural Science. 